Q3. Artículos de Investigación 
ANALYSIS OF THE SUSTAINABILITY

OF REGIONAL NATURAL MATERIALS

FOR THE DEUELOPMENT OF

CONSTRUCTION TECHNOLOGIES

THAT GENERATE ENERGY

\section{EFFICIENCY ON NEA'S BUILDING}

KEYWORDS

Sustainability; efficiency; guideline

ABSTRACT

The work summarizes an analysis of the regulations, inside the region and at the national level, that show the current situation of sustainability and energy efficiency of buildings. Besides, it exposes existing and current parameters and guidelines that helped to result in a proposal of possible indicators to be incorporated into the regulations of the region. On the other hand, with greater importance in terms of materiality, research carried out in the region was taken into account that put the magnifying glass on energy saving with an emphasis on wood: an abundant, economical and energy efficient resource in the NEA. 


\title{
ANÁLISIS DE LA SUSTENTABILIDAD DE LOS MATERIALES NATURALES REGIONALES PARA EL DESARROLLO DE TECNOLOGIAS CONSTRUCTIUAS QUE GENEREN EFICIENCIA ENERGÉTICA EN LA EDIFICACIÓN DEL NEA
}

Joaquín JIMÉNEZ; Jacobo, Guillermo

joaquinjimenezpach@gmail.com gjjacobo@arq.unne.edu.ar

\begin{abstract}
- Estudiante avanzado y becario de la Facultad de Arquitectura y Urbanismo, en la cátedra de Estructuras II, FAU-UNNE. - Investigador SGCyT-UNNE y CIN. Profesor titular (FAU-UNNE).
\end{abstract}

PALABRAS CLAUE

Sustentabilidad; eficiencia; lineamiento.

RESUMEN

El trabajo resume un análisis de las normativas, dentro de la región y a nivel nacional, que muestran la situación actual de la sustentabilidad y la eficiencia energética de los edificios. Además, expone parámetros y lineamientos existentes y vigentes que ayudaron a dar como resultado una propuesta de indicadores posibles para incorporar en las normas de la región. Por otra parte, con mayor importancia en cuanto a la materialidad, se tomaron en cuenta investigaciones realizadas en la región que ponen la lupa sobre el ahorro energético haciendo énfasis en la madera: un recurso abundante, económico y energéticamente eficiente en el NEA. 


\section{OBJETIUOS}

\section{Objetivos generales}

1. Analizar la cuestión de la sustentabilidad de los materiales naturales regionales para la aplicación en la arquitectura y la construcción, tal y como se detecte en las normativas respecto de la edificación vigentes en las ciudades de Resistencia y Corrientes (Reglamento General de Construcciones, en Resistencia, y Código de Edificación, en Corrientes).

2. Realizar un diagnóstico según el análisis anterior, con base en el cual desarrollar y proponer posibles criterios e indicadores locales de tecnologías constructivas para la edificación sustentable, que constituyan la base de lineamientos de posible incorporación en las normativas de la edificación analizadas de Resistencia y Corrientes (que contemplen factores urbanos, socio-culturales, climáticos y económicos locales y regionales)

\section{Objetivos particulares}

1) Estudiar parámetros de evaluación de sustentabilidad de materiales regionales para el desarrollo de tecnologías constructivas, para la edificación energéticamente eficiente en el NEA.

2) Detectar en los sistemas de evaluación de sustentabilidad sus enfoques, acercamientos al tema de la evaluación edilicia y su incorporación en el proceso proyectual, así como su grado de aplicabilidad a la evaluación de la arquitectura local de Resistencia y Corrientes.

3) Analizar las normativas vigentes respecto de la edificación en las ciudades de Resistencia y Corrientes (Reglamento General de Construcciones, en Resistencia, y Código de Edificación, en Corrientes).

4) Considerar los condicionantes $y$ determinantes de la realidad local de la construcción formal e informal de Resistencia y Corrientes, a fin de detectar los distintos niveles y escalas de intervención.

5) Diagnosticar la calidad de las tecnologías regionales y su impacto ambiental, desde el punto de vista de la sustentabilidad, tal y como se detecte en el análisis de las normativas referidas.

6) Proponer parámetros e indicadores locales de edificación sustentable y eficienteenergéticamente, en distintos niveles y escalas de intervención, en función del diagnóstico anterior, que apunten a una mejora de los aspectos críticos y/o no contemplados en las normativas edificatorias analizadas.

\section{INTRODUCCIÓN}

El debate sobreedificación sustentable se encuentra centrado en eficiencia y comportamiento estableciendo, a pesar de sus limitaciones en tiempos de experimentación, un punto de partida valorable y medible en el proceso de desarrollo de criterios e instrumentos para calificar y evaluar la calidad ambiental de edificios e implementar la certificación de la producción de hábitat. La edificación sustentable promueve diversos beneficios que se extienden más allá de su participación en el mejoramiento de las condiciones ambientales y la mitigación del impacto ambiental, dado que representan el establecimiento de un nuevo orden de los principios básicos de diseño en todas y cada una de sus escalas. Dichos principios se fundamentan en sistemas y ciclos naturales, una mayor dependencia en recursos locales, particularmente para la generación, distribución y uso de energía y agua, con dimensión social y proyección a futuro (De Schiller et al., 2003).
La implementación de sistemas de control ambiental mediante etiquetado edilicio ha tomado fuerza en el nuevo siglo en varios países industrializados, e impone principios de arquitectura sustentable como importante medida contra los efectos negativos que provocan el cambio climático y las crisis energéticas (Blasco Lucas, 2008).

En los países industrializados, los sistemas de evaluación y certificación de sustentabilidad de edificios se han convertido en una herramienta para lograr valor agregado y posicionamiento en el mercado inmobiliario. El desarrollo de este tipo de instrumentos contribuye a un proceso de diseño de edificios cada vez más consciente y responsable en aspectos de sustentabilidad. Repetir esta práctica en Argentina y más concretamente en la región Nordeste (NEA) requiere contar con un sistema de evaluación de edificios que tenga el respaldo y la obligatoriedad de aplicación dados por el aval normativo legal, que se ajuste además a las condiciones y posibilidades sociales, ambientales y económicas locales. El proceso de desarrollo e implementación de estos sistemas fue el eje principal de redes de investigación y desarrollo con particular énfasis en la última década.

Actualmente Argentina atraviesa una crisis energética y demanda urgentes medidas en todos los campos posibles de acción para paliarla, entre los cuales el propio de la arquitectura sustentable debería jugar un rol privilegiado. En este marco, cabe destacar que la industria de la construcción aporta el 5 \% del PBI (2005) y ha sido la principal impulsora de 
la recuperación del empleo después de la terrible crisis socioeconómica del año 2002 (INDEC, 2001). Aun contando con todos estos antecedentes que indican la pertinencia e importancia del área construida en lo referido al impacto que produce en el balance energético-ambiental del país, las decisiones que se toman para lograr una mayor eficiencia están enfocadas en otros sectores (Blasco Lucas, 2008).

Es importante reconocer la escasa conciencia que existe respecto de los beneficios de la construcción energéticamente eficiente y de bajo impacto, tanto económicos como ambientales. Otro tanto sucede en el ámbito profesional e institucional, dado que la legislación edilicia de la gran mayoría de países de la región no incluye la eficiencia energética en edificios ni presenta exigencias de calidad térmica. Por lo tanto, la construcción en la región no incorpora adecuados niveles de aislamiento térmico de forma obligatoria (De Schiller et al., 2003).

PRIMERA ETAPA: ANÁLISIS Y DIAGNÓSTICO

\section{Normativas y la sustentabilidad}

\section{Análisis de normativas de la región NEA}

En primer lugar, se realizó la recopilación, lectura y análisis de las normativas existentes dentro de la región del NEA (ciudad de Resistencia, Chaco y ciudad de Corrientes, Corrientes). Los datos brindados en diferentes fuentes, como libros, revistas en la biblioteca de la Facultad de Arquitectura y Urbanismo como también publicaciones en Internet y antecedentes de becas de investigación realizados por otros becarios fueron tomados en cuenta para esta primera etapa. A partir de esto, se efectuaron comparaciones de normativas vigentes en otros países, como Alemania, que poseen exigencias en cuanto a la sustentabilidad y eficiencia energética edilicia, y también normativas existentes dentro del país, como Buenos Aires y Rosario, que puedan servir de base para la implementación dentro del NEA. Con base en estas comparaciones, las exigencias son mínimas o casi nulas dentro de la región, aún más teniendo en cuenta la zona bioclimática a la que pertenecen las ciudades de Resistencia y Corrientes. Si bien las Normas IRAM exigen niveles de transmitancia térmica mínimos para cada zona bioambiental del país, estos no son respetados o tomados en cuenta en las construcciones dentro de la región, lo que genera significativos valores de ganancias y pérdidas de temperatura.

En el marco normativo existente en la región del NEA (comprendida por la ciudad de Corrientes y Resistencia) no se plantean las cuestiones sustentables de los materiales regionales empleados en la construcción de edificios, como por ejemplo utilización, cálculos, determinación de coeficientes mínimos y máximos, tratamientos, etc., que generen eficiencia energética. Estas normativas plantean cuestiones generales de la construcción, tales como espesores mínimos y consideraciones generales en cuanto a la transmitancia térmica. La normativa vigente en la ciudad de Resistencia (Reglamento General de Construcciones) plantea, dentro de la cuestión de habitabilidad, las condiciones que deben reunir las viviendas sugiriendo la aplicación de las Normas IRAM vigentes. Sin embargo, no exige el cumplimiento estricto de estas ni la realización y presentación de los cálculos correspondientes dentro de la institución respectiva que habilita la ejecución de la obra.

\section{Análisis de las normativas en Argentina}

A nivel nacional, distintas ciudades, como Buenos Aires y la ciudad de Rosario, en Santa Fe, proponen normativas que definen un diseño sustentable a la hora de hablar de cuestiones edilicias. En la provincia de Buenos Aires existe la Ley 13059 de acondicionamiento higrotérmico de edificios y también existe un manual realizado con la recopilación y selección elaborada por especialistas, diseñado y concebido para orientar la aplicación de dicha ley. Este manual posee dos partes: la primera realiza el desarrollo de los conceptos de sustentabilidad aplicados a la construcción de edificios, la habitabilidad higrotérmica, el calor, la humedad y el confort higrotérmico; y en la segunda parte, desarrolla las normas IRAM de aislamiento térmico en edificios. Este manual genera una dinámica a la hora de entender las cuestiones planteadas en la ley y hace mucho más fácil la comprensión de las Normas IRAM realizando una síntesis enfocada en el sector bioclimático al que pertenece la provincia de Buenos Aires.

Por otra parte, y a efectos de la Ley provincial 13059, el Código de Edificación de la Ciudad Autónoma de Buenos Aires y el Plan Urbano Ambiental (Ley 2930) constituyen un marco legal importante en cuanto a la sustentabilidad edilicia. Dicho código de edificación plantea el diseño sustentable a proyectos de gran escala, como lo son construcciones nuevas o ampliaciones mayores 
a $1000 \mathrm{~m}^{2}$. Esta normativa exige diferentes puntos o características de diseño que se deben cumplir a la hora de proyectar. Estos puntos son la ganancia solar, la protección solar, la ventilación natural, el aislamiento térmico de la envolvente, la transmitancia térmica, el factor solar, los techos fríos, el confort acústico y visual, la calidad del aire interior, el uso del agua, el consumo energético, los jardines verticales, techos verdes, el uso del agua de lluvia y también la ralentización del agua de lluvia para sectores inundables. Sin embargo, no solo plantea estas cuestiones - verificables a la hora de terminar la obra-, sino que además plantea la gestión ambiental en el proceso constructivo. En este apartado, determina la reducción del impacto ambiental de la actividad constructiva para orientar a un manejo sustentable de las obras, sus procesos y recursos haciendo hincapié en la cumplimentación de los requisitos de gestión ambiental del proceso constructivo fijado en el organismo competente.

En cuanto a la normativa vigente a partir del año 2011 en la ciudad de Rosario, provincia de Santa Fe, contiene una sección específica donde se consideran los aspectos higrotérmicos y la demanda energética en la construcción que se aplica a toda obra nueva, pública o privada, y modificaciones, reformas o rehabilitaciones que superen los $500 \mathrm{~m}^{2}$ de superficie útil. Para ello plantea la verificación del proyecto en cuanto a las Normas IRAM vigentes en Argentina: control de las condiciones de habitabilidad (IRAM 11605), condensaciones superficiales e intersticiales (en el interior de los paramentos), control indirecto de la demanda energética de los edificios en calefacción (IRAM 11604) y en refrigeración (IRAM 11659/1 y /2). Además de la verificación del proyecto, también plantea la verificación de la construcción, en donde existe un apartado específico que exige características de los productos y el control de recepción en obra sobre la base de las normas nacionales, como lo son las Normas IRAM 11601 (conductividades térmicas de cada material), y si los materiales utilizados no están en la lista, exige las pruebas en organismos certificados de acuerdo con las normas IRAM 11559 (para métodos de ensayo, determinación de la resistencia térmica y propiedades conexas en régimen estacionario. Método de la placa caliente con guarda) y 1860 (método de ensayo de las propiedades de transmitancia térmica en régimen estacionario mediante el aparato de medición del flujo de calor). También determina un control durante el proceso de construcción de manera frecuente, en el que se comprobará la aplicación de las condiciones mínimas exigibles en la norma y propuestas en el proyecto y determina que toda modificación realizada durante la ejecución de la obra debe quedar documentada sin que en ningún caso dejen de cumplirse las condiciones mínimas señaladas en la normativa.

\section{Normas IRAM}

Es de suma necesidad el estudio de los parámetros y lineamientos de diseño tomados por el Instituto Argentino de Normalización y Certificación en sus denominadas Normas IRAM. Estas normas proponen un abanico de estudios, parámetros y lineamientos de diseño que son una herramienta de base para el confort dentro de la construcción. Es vital tener en cuenta y aplicar sus normas para la región del NEA, comprendida, en este trabajo de investigación, por las ciudades de Corrientes y Resistencia.

\section{Diseño sustentable: \\ lineamientos y parámetros de valoración}

Otra de las ciudades que va encaminada hacia el diseño sustentable es Córdoba, que posee un sistema denominado ese (Etiquetado de Sustentabilidad Edilicia), elaborado y registrado por el Instituto de Arquitectura Sustentable (IAS) del Colegio de Arquitectos de Córdoba. Es un sistema integral de auditoría y evaluación de performances de variables sustentables en edificios, tanto en etapa de proyecto como en los ya existentes. Este sistema pretende mejorar la eficiencia integral sustentable del diseño, la construcción y el uso efectivo de los edificios teniendo en cuenta el factor medioambiental y aspectos económicos y sociales para generar un equilibrio en el marco del desarrollo sustentable.

El arquitecto Edgardo F. Suárez sintetiza los parámetros tenidos en cuenta para la etiquetación de sustentabilidad edilicia en la provincia de Córdoba de la siguiente manera:

\section{DISTRIBUCIÓN RELATIVA DE PUNTAJES POR ETIQUETA}

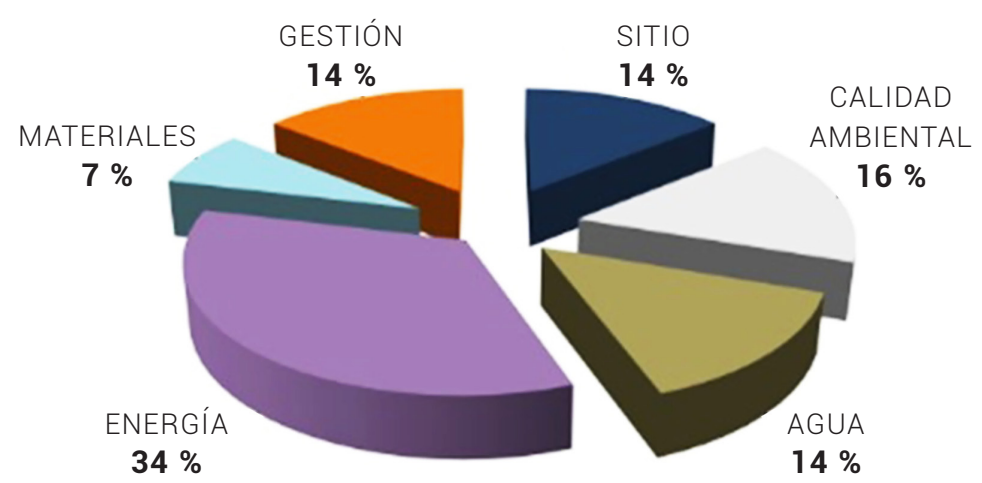




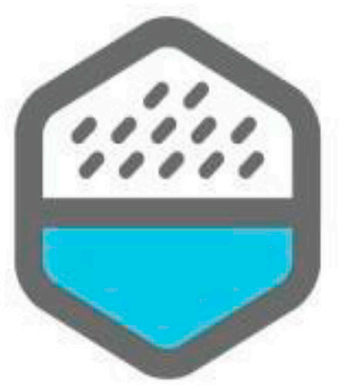

\section{AGUA}

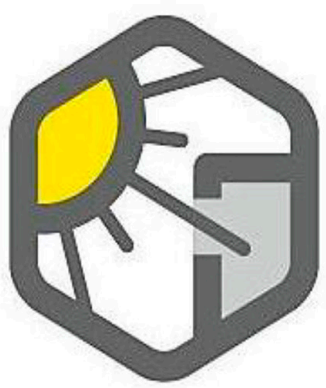

CALIDAD AMBIENTAL
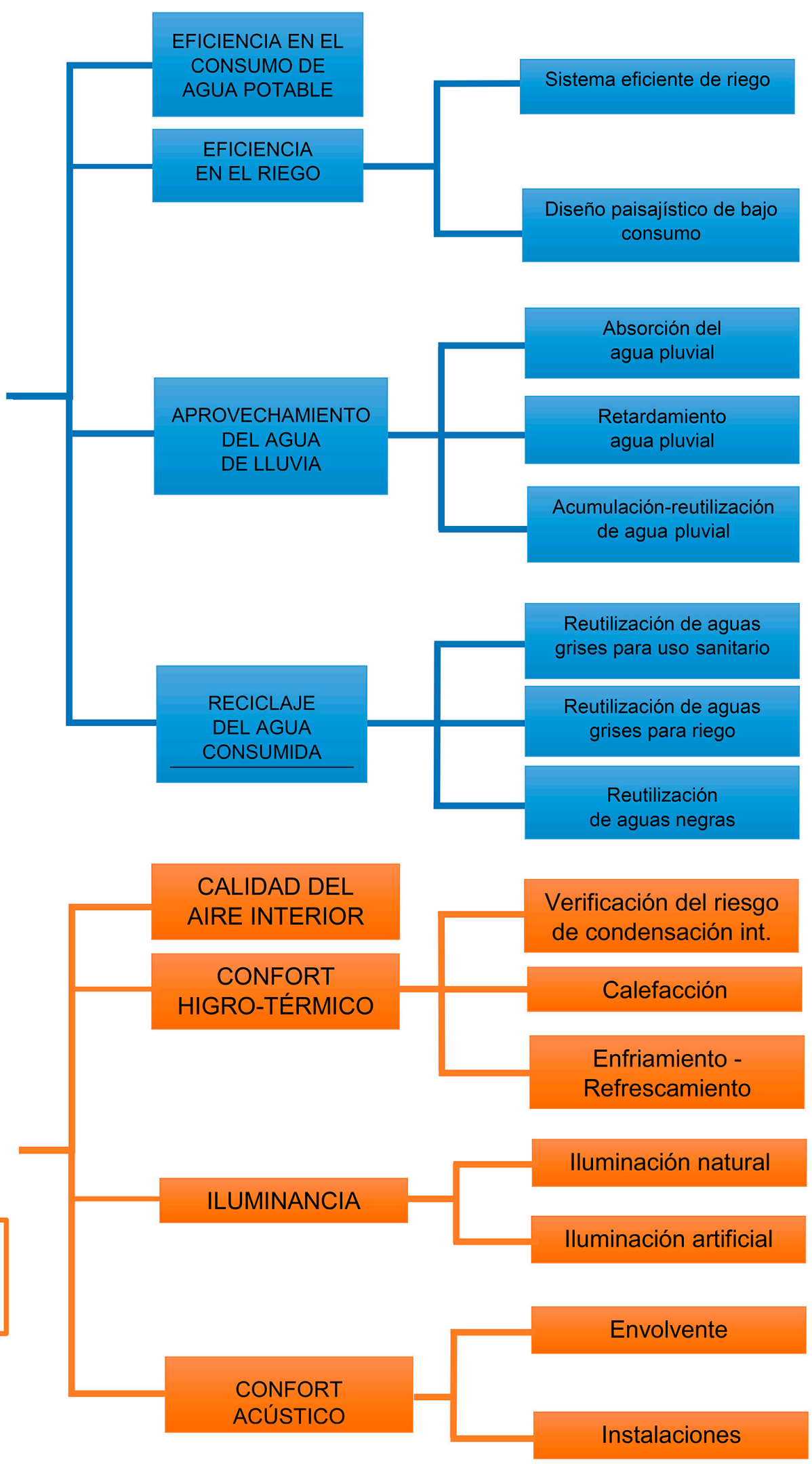

Figura 1. Cuadro síntesis de los parámetros del sistema eSe. Fuente: Edgardo F. Suárez (2017) 


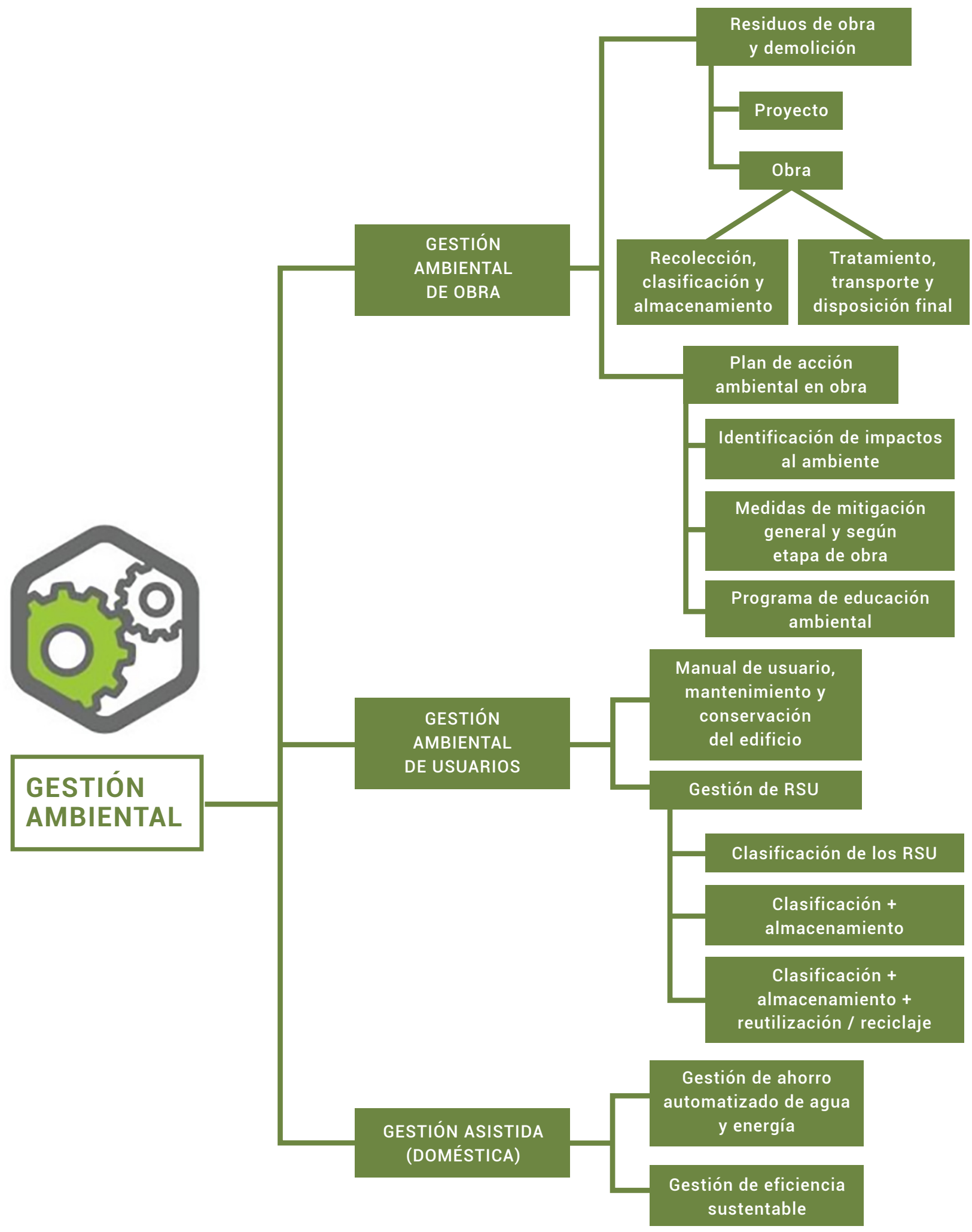

Figura 1. Cuadro síntesis de los parámetros del sistema eSe. Fuente: Edgardo F. Suárez (2017) 


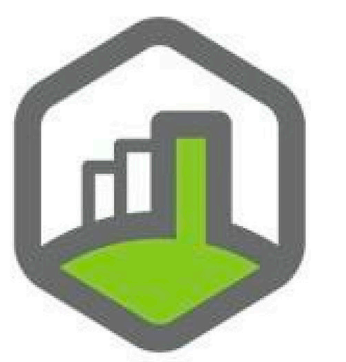

\section{SITIO}

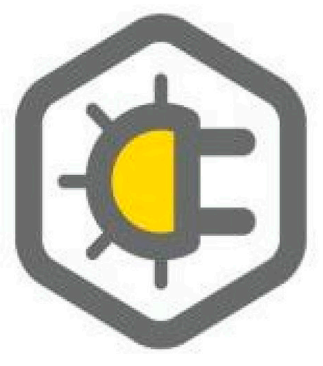

\section{ENERGÍA}

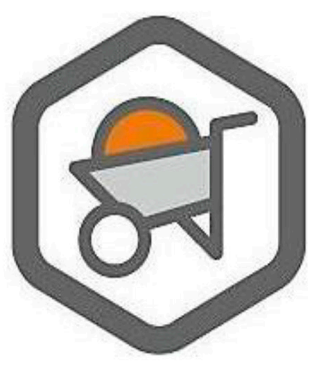

MATERIALES

\section{ISLA DE CALOR URBANA}

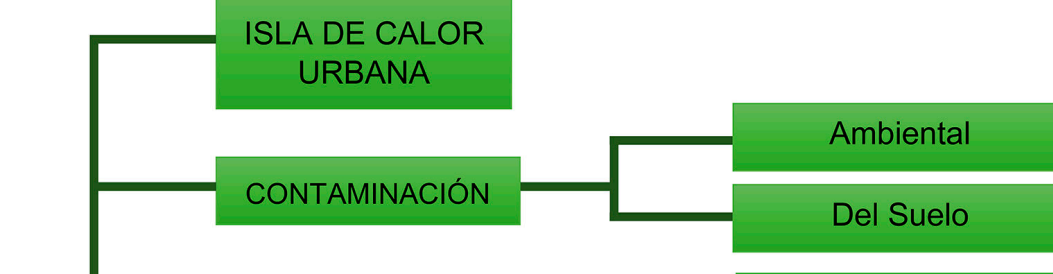

Accesibilidad al transporte

Accesibilidad al equipamiento básico

CONECTIVIDAD Y SERVICIOS

Estacionamiento de vehículo alternativo

Consideración ambiental del sitio

Conservación y recuperación de la cobertura vegetal

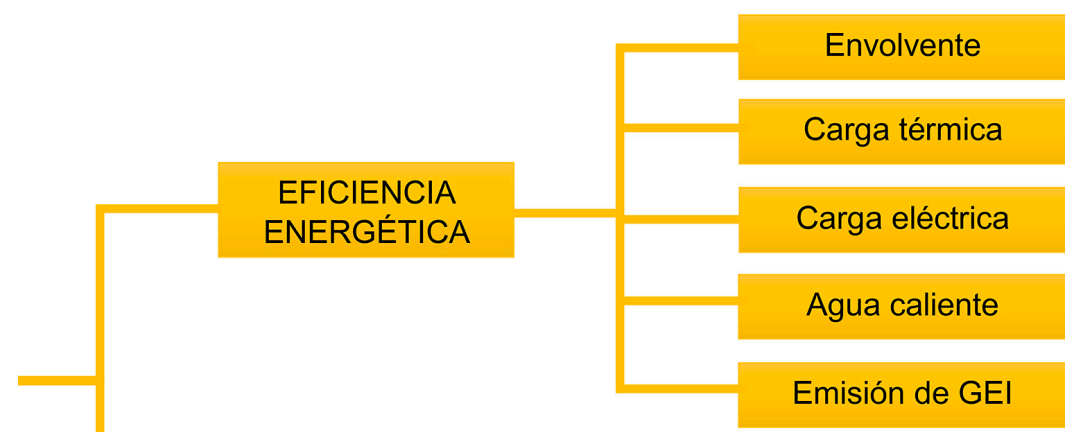

\section{AUTOGENERACIÓN} ENERGÉTICA

Reutilización de edificio existente

Reutilización de materiales

MATERIALIDAD

CERTIFICADO/BAJO

MANTENIMIENTO

Figura 1. Cuadro síntesis de los parámetros del sistema eSe. Fuente: Edgardo F. Suárez (2017) 
A nivel regional, se han realizado trabajos que abordan el tema de los lineamientos e indicadores para la sustentabilidad edilicia dentro de la región. Carla Romano Pamies, Herminia M. Alías y Guillermo J. Jacobo (2018) en su trabajo de investigación "Lineamientos e indicadores para la valoración de la sustentabilidad edilicia, de factible incorporación a las normativas de edificación vigentes en Resistencia y Corrientes" analizaron las certificaciones mediante LEED (USA) y BREEAM (Reino Unido). Esta última fue estudiada por su gran utilización a nivel internacional. Estos autores, luego de los análisis de estos certificados, concluyen:

Ambas metodologías tienen amplia influencia en gran parte del mundo y generan información técnica objetiva respecto al nivel de sostenibilidad de un edificio (otorgando distintos tipos de certificados), información que eventualmente aporta transparencia al mercado inmobiliario y fomenta las inversiones en ahorro de energía y reducción de emisiones de CO2 (GEES, Cátedra UNESCO de sostenibilidad, UPC, 2011) que además contribuye a mejorar los costos de operacióny el aprovechamiento de los recursos consumidos por el edificio.

De esta manera, y en su etapa de propuesta y definición de indicadores locales, elaboraron un listado de lineamientos, primero de manera general y luego profundizando cada uno de ellos para detectar su campo de aplicación específico; así, confeccionaron un sistema de planillas a modo de instrumento para la valoración y puntuación. Los indicadores clasificados por aspectos están valorados de forma numérica, se determinan en cinco niveles diferentes que representan el grado de incidencia de cada indicador. Las valoraciones van del 0 al 100 a modo de porcentajes. La planilla arrojó un resultado de 2500 referido a un valor estimado de sustentabilidad "óptima", ya que un edificio debería rondar ese valor o superarlo.

\section{Diseño sustentable: la materialidad}

Actualmente, las viviendas de interés social son un punto vulnerable dentro de las ciudades porque se deben minimizar los costos de mantenimiento e inversión para garantizar adecuadas condiciones de habitabilidad. En cuanto a la materialidad regional, el Arq. Guillermo Jacobo y la Arq. Herminia Alías (2007) han realizado un trabajo acerca de la construcción sostenible dentro de la región del Nordeste Argentino desde el punto de vista de esta área crítica del diseño en las ciudades.

Dentro del trabajo, además, se efectuó un análisis de tipologías de viviendas de interés social difundidas e implementadas masivamente en las provincias de Corrientes y Chaco. Se analizaron los esquemas de estas tipologías, se establecieron características principales de cada una y se eligieron las de mayor representatividad. Los aspectos tomados fueron clasificados en dos grupos: 1) aspectos funcionales y morfológicos edilicios: forma del edificio, orientación, etc., y 2) aspectos tecnológicos-constructivos: desagregación de los componentes de la envolvente edilicia, muros, cubiertas, etc. La indagación de las variables definidas recabó información sobre tres cuestiones centrales para el trabajo:
- La situación higrotérmica de los componentes de la envolvente de las unidades de análisis.

- Los consumos de energía eléctrica para el acondicionamiento térmico para el confort dentro de cada unidad de análisis, según características higrotérmicas de sus envolventes (trabajo centrado en los muros) y según otras variables (orientaciones, implantación, forma).

- La estimación de la energía total involucrada en $1 \mathrm{~m}^{2}$ de panelería forestal del NEA en comparación con $1 \mathrm{~m}^{2}$ de muro de mampostería de ladrillo común de fabricación artesanal fabricada en los obrajes de Bañado Norte de la ciudad de Corrientes. No solo la realización, sino en la etapa completa del ciclo de vida de estos elementos (extracción de materia prima, transporte, procesamiento, producción, puesta en obra, uso durante la vida útil y disposición final). Se realizaron comparaciones de perfiles ambientales de los materiales empleados en un metro cuadrado de mampostería de ladrillo común (1) y un metro cuadrado de panelería de madera de pino (2).

En resumen:

1. Requiere otros materiales, como el cemento, que posee un gran impacto ambiental en varias categorías comparadas. Impacta sobre el potencial de calentamiento global (efecto invernadero), porque posee grandes emisiones de $\mathrm{CO} 2$ durante la cocción de los ladrillos y también en el potencial de acidificación (que posee propiedades de un ácido), tanto durante el procesamiento y producción y en la fase de uso, que se estima de cuarenta años.

2. Posee un perfil ambiental más benigno comparativamente con el muro de mampostería de ladrillo 
común. Sus efectos negativos se originan en el proceso de secado e impregnación de la madera.

Los resultados de la energía (energía eléctrica) necesaria para el confort según la simulación computacional (SimaPro Demo) que utilizaron para realizar este análisis pudieron ayudar a la definición de factores decisivos que influyen fuertemente durante la fase de uso de las viviendas:

1. Material constitutivo de muros y cubiertas

2. Orientación.

3. Morfología.

Concluyeron entonces:

\author{
Mediante las herramientas apli- \\ cadas se pudo demostrar que la \\ construcción en madera repre- \\ senta una alternativa construc- \\ tiva más eficiente, económica y \\ benigna desde el punto de vista \\ ambiental que la construcción \\ tradicional (mediante la técnica \\ del mampuesto de fabricación \\ local artesanal) de viviendas de \\ interés social en el NEA. Ha sido \\ corroborada en alto grado en \\ base a los resultados obtenidos \\ a través de los procedimientos \\ aplicados (simulación compu- \\ tacional de consumo de energía \\ para el confort de viviendas, \\ análisis de componentes de la
}

envolvente mediante normativa IRAM de habitabilidad higrotérmica y aplicación introductoria y aproximada al ACV, según norma ISO 14.040) (Alías y Jacobo, 2007).

\section{SEGUNDA ETAPA}

\section{Propuesta y definición de indicadores}

Sobre la base de lo desarrollado en la primera etapa de análisis y diagnóstico, pudimos definir distintos parámetros e indicadores de sustentabilidad. El siguiente cuadro sintetiza algunos de ellos.

\begin{tabular}{|c|c|c|c|}
\hline FIGURA 2 & \multicolumn{3}{|c|}{$\begin{array}{l}\text { Cuadro síntesis de parámetros e indicadores } \\
\text { posibles para la región del NEA }\end{array}$} \\
\hline ASPECTO & PARÁMETROS & ¿QUÉ IMPLICA? & $\begin{array}{l}\text { NORMATIVA } \\
\text { DE CONSULTA } \\
\text { I APLICACIÓN }\end{array}$ \\
\hline $\begin{array}{l}\text { LUGAR } \\
\text { ENERGÍA } \\
\text { MATERIALIDAD }\end{array}$ & $\begin{array}{l}\text { 1. Implantación } \\
\text { 2. Accesibilidad } \\
\text { a servicios } \\
\text { 3. Vegetación }\end{array}$ & $\begin{array}{l}\text { 1. Orientación, consideraciones del clima en } \\
\text { diseño y forma, cumplimiento del FOS y FOT } \\
\text { 2. Agua, cloaca, transporte público. }\end{array}$ & $\begin{array}{l}\text { Norma IRAM } \\
11603 .\end{array}$ \\
\hline \multirow[t]{3}{*}{ AGUA } & $\begin{array}{l}\text { 1. Envolvente } \\
\text { 2. Autogenera- } \\
\text { ción de energía }\end{array}$ & $\begin{array}{l}\text { 1. Utilización de protecciones solares, } \\
\text { verifica-ción de puentes térmicos, fachadas } \\
\text { con doble piel, utilización de cámaras de } \\
\text { aire ventiladas, techos sombra, etc. } \\
\text { 2. Utilización de energía alternativa para abaste- } \\
\text { cimiento propio (solar, eólica, biomasa, etc.) }\end{array}$ & $\begin{array}{l}\text { Norma IRAM } \\
11601 \text { y } 11604 . \\
\text { Leyes naciona- } \\
\text { les de energías } \\
\text { renova-bles. }\end{array}$ \\
\hline & $\begin{array}{l}\text { 1. Elección de } \\
\text { mate-riales } \\
\text { 2. Proyección de } \\
\text { "arquitectura verde" }\end{array}$ & $\begin{array}{l}\text { 1. Materiales regionales, materiales } \\
\text { con coeficien-tes de transmitancia } \\
\text { térmica baja, verificación de } \\
\text { condensaciones, inercia térmica, etc. } \\
\text { 2. Proyección de muros verdes, techos } \\
\text { verdes, terrazas verdes, etc. }\end{array}$ & $\begin{array}{l}\text { Norma IRAM } \\
11601 \text { y } 11604\end{array}$ \\
\hline & $\begin{array}{l}\text { 1. Aprovechamiento } \\
\text { del agua de lluvia } \\
\text { 2. Reutilización de } \\
\text { agua consumida } \\
\text { 3. Eficiencia } \\
\text { en el riego }\end{array}$ & $\begin{array}{l}\text { 1. Retardamiento del agua pluvial, acumulación } \\
\text { y reutilización del agua de lluvia. } \\
\text { 2. Reutilización de aguas grises } \\
\text { para uso sanitario y/o riego. } \\
\text { 3. Utilización de sistemas eficientes de riego, } \\
\text { diseño de paisajismo de bajo consumo. }\end{array}$ & $\begin{array}{l}\text { Régimen } \mathrm{Na-} \\
\text { cional de Reúso } \\
\text { para Aguas } \\
\text { Residuales. }\end{array}$ \\
\hline
\end{tabular}

Fuente: elaboración propia 
Podemos sintetizar la Norma IRAM 11603 en los siguientes aspectos que

hay que considerar dentro del NEA

1. Cumplimiento estricto de las siguientes condiciones planteadas en el punto 5 "Recomendaciones generales en el diseño" de la misma norma:

- Respetar las siguientes orientaciones:

\begin{tabular}{|c|c|c|c|c|}
\hline \multirow{3}{*}{$\begin{array}{r}\text { Figura } 3 . \\
\text { Orientaciones } \\
\text { que reciben } \\
\text { dos horas de } \\
\text { asoleamiento, } \\
\text { orientaciones } \\
\text { favorables } \\
\text { yóptimas y } \\
\text { orientaciones } \\
\text { con protección } \\
\text { solar necesaria. } \\
\text { Fuente: Norma } \\
\text { IRAM } 11603\end{array}$} & $\begin{array}{l}\text { ZONAS } \\
\text { BIOAYB. }\end{array}$ & $\begin{array}{l}\text { ORIEHT C/PROTBC. } \\
\text { SOLAR AECESARIA }\end{array}$ & $\begin{array}{l}\text { ORIFET DONDE SB } \\
\text { RBCIBES } 2 \text { HS ASOL. }\end{array}$ & $\begin{array}{l}\text { ORIENT FAVORABLES } \\
\text { Y OPTIMAS }\end{array}$ \\
\hline & 总 & & & OiN \\
\hline & 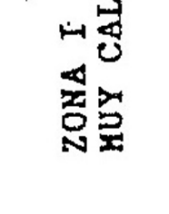 & $\sqrt{5}$ & is & $\cdot$ \\
\hline
\end{tabular}

- Colores claros en paredes exteriores y techos.

- Gran aislación térmica en techos y en las paredes orientadas al este y al oeste.

- Bajo todos los conceptos, deben estar todas las superficies protegidas de la radiación solar

- La ventilación cruzada de la vivienda para disminuir el "disconfort".

- La existencia de los espacios semicubiertos (galerías, balcones, terrazas, patios)

- Deberá considerarse la necesidad de aprovechar los vientos dominantes y la creación de alta y baja presión que aumente la circulación del aire.

\section{Cumplir, si existe posibilidad dentro} del proyecto, los siguientes ítems: - El eje mayor de la vivienda será, preferentemente, este-oeste.

- Para las ventanas, si es posible, no orientarlas al este o al oeste y minimizar su superficie.

- Sería sumamente conveniente que los espacios semicubiertos puedan ser protegidos de los insectos, aunque la necesidad de mosquiteros implica, contrariamente, una sensible reducción de la ventilación. Analizar qué sectores pueden ser protegidos de los insectos.

- Deberá tenerse en cuenta la nece- sidad de minimizar las superficies que miren al oeste y al este.

\section{Radiación solar}

Para las regiones cálidas, las orientaciones térmicamente favorables coinciden con las de mínimo asoleamiento (dos horas de asoleamiento).

\section{Asoleamiento en invierno}

Las recomendaciones mínimas de asoleamiento invernal de esta norma facilitan la verificación y aseguran niveles mínimos del aporte de energía solar, tomando en cuenta la variación de radiación directa según la altura del sol, la transmisión de la radiación a través de vidrios según el ángulo de incidencia y la relación entre el costo del proyecto y los beneficios del asoleamiento.

\section{Requisitos de verificación}

En locales ubicados al norte de la latitud $47^{\circ}$ sur, cada vivienda deberá cumplir con los siguientes requisitos: a) un mínimo de dos horas al sol directo en el solsticio de invierno (23 de junio) a través de las ventanas de por lo menos la mitad de los locales habitables;

b) solamente se acepta el período de asoleamiento cuando la altura del sol es mayor que $10^{\circ}$;

c) no se considera el asoleamiento cuando el ángulo de incidencia es mayor que $67,5^{\circ}$.

En la figura 4 se indican las orientaciones que permiten obtener el asoleamiento mínimo en zonas residenciales de media y baja densidad. En los casos en que la altura angular de edificios u otros obstáculos es mayor que $20^{\circ}$, es necesario verificar el asoleamiento utilizando métodos gráficos o simulaciones en escala. En conjunto de viviendas multifamiliares, se acepta hasta un $10 \%$ de unidades sin asoleamiento, siempre y cuando el agrupamiento resultante logre beneficios bioambientales, tales como protección del viento en los espacios exteriores o formas compactas que disminuyan las pérdidas de calor.

En edificios que aprovechen la radiación solar a través de sistemas solares pasivos, es necesario obtener por lo menos seis horas de asoleamiento para optimizar la captación de energía. En este caso, los niveles de aislación térmica del edificio deberán ser superiores a las exigencias de la norma IRAM 11604 


\begin{tabular}{|c|c|c|c|c|}
\hline \multirow{2}{*}{ LATITUD SUR } & \multirow{2}{*}{ Fecha de verificación } & \multicolumn{2}{|c|}{ Orientaciones respecto del Norte } & \multirow{4}{*}{$\begin{array}{l}\text { Figura } 4 . \\
\text { Orientaciones } \\
\text { de aberturas } \\
\text { que permi- } \\
\text { ten obtener } \\
\text { dos horas de } \\
\text { asoleamiento } \\
\text { mínimo. Fuen- } \\
\text { te: Normas } \\
\text { IRAM } 11603\end{array}$} \\
\hline & & Con edificación ${ }^{1)}$ & Sin edificación ${ }^{2)}$ & \\
\hline $\begin{array}{l}22^{\circ} \text { a } 28^{\circ} \\
28^{\circ} \text { a } 33^{\circ} \\
33^{\circ} \text { a } 38^{\circ} \\
38^{\circ} \text { a } 42^{\circ} \\
42^{\circ} \text { a } 47^{\circ} \\
47^{\circ} \text { a } 52^{\circ} \\
52^{\circ} \text { a } 55^{\circ}\end{array}$ & $\begin{array}{c}23 \text { de junio } \\
23 \text { de junio } \\
23 \text { de junio } \\
23 \text { de junio } \\
23 \text { de junio } \\
15 \text { de agosto ó } 30 \text { de abril } \\
15 \text { de agosto ó } 30 \text { de abril }\end{array}$ & $\begin{array}{l}260^{\circ} \text { a } 110^{\circ} \\
263^{\circ} \text { a } 96^{\circ} \\
267^{\circ} \text { a } 93^{\circ} \\
270^{\circ} \text { a } 90^{\circ} \\
280^{\circ} \text { a } 80^{\circ} \\
270^{\circ} \text { a } 90^{\circ} \\
280^{\circ} \text { a } 80^{\circ}\end{array}$ & $\begin{array}{c}- \\
- \\
- \\
- \\
- \\
- \\
270^{\circ} \text { a } 90^{\circ}\end{array}$ & \\
\hline \multicolumn{4}{|c|}{$\begin{array}{l}\text { 1) Terrenos con edificación u otros obstáculos típicos de zonas urbanas y suburbanas, con una altura angular menor que } 20^{\circ} \text {. } \\
\text { Cuando los obstáculos son mayores que } 20^{\circ} \text { es recomendable realizar la verificación con métodos especíicos. } \\
\text { 2) Terrenos sin edificación, árboles u otros obstáculos que disminuyen el asoleamiento. Ángulo máximo de obstáculos igual } \\
\text { a } 10^{\circ} \text {. }\end{array}$} & \\
\hline
\end{tabular}

5. Recomendaciones sobre protecciones solares

Se aconseja para las zonas bioambientales I a IV y para las orientaciones SO-O-NO-N-NE-E-SE el uso de sistemas de protección solar, como por ejemplo parasoles horizontales y verticales, cortinas de enrollar de color claro. Se recomienda el uso de los parasoles, para cuyo cálculo se aconseja el empleo de la carta solar en la definición de las medidas adecuadas (figura "orientación con protección solar necesaria)

\section{Evaluación de los microclimas Clima cálido}

Las distribuciones edilicias abiertas atenúan el efecto de isla caliente y favorecen la ventilación. Por este motivo, resultan favorecidas las ubicaciones a barlovento de cualquier obstáculo (sierra, zona boscosa).

La distribución edilicia al pie de la pendiente en los valles evita el marcado calentamiento diario y aprovecha la brisa de pendiente durante las noches. Por su efecto atemperador, la cercanía a masas de agua resulta beneficiosa, como en la zona fría.

Como ejemplo, se mostrarán a continuación recomendaciones tecnológicas para la región del NEA sobre la base de estudios realizados por el Arq. Jacobo Guillermo y Arq. Alías, Herminia (Revista Arquisur).

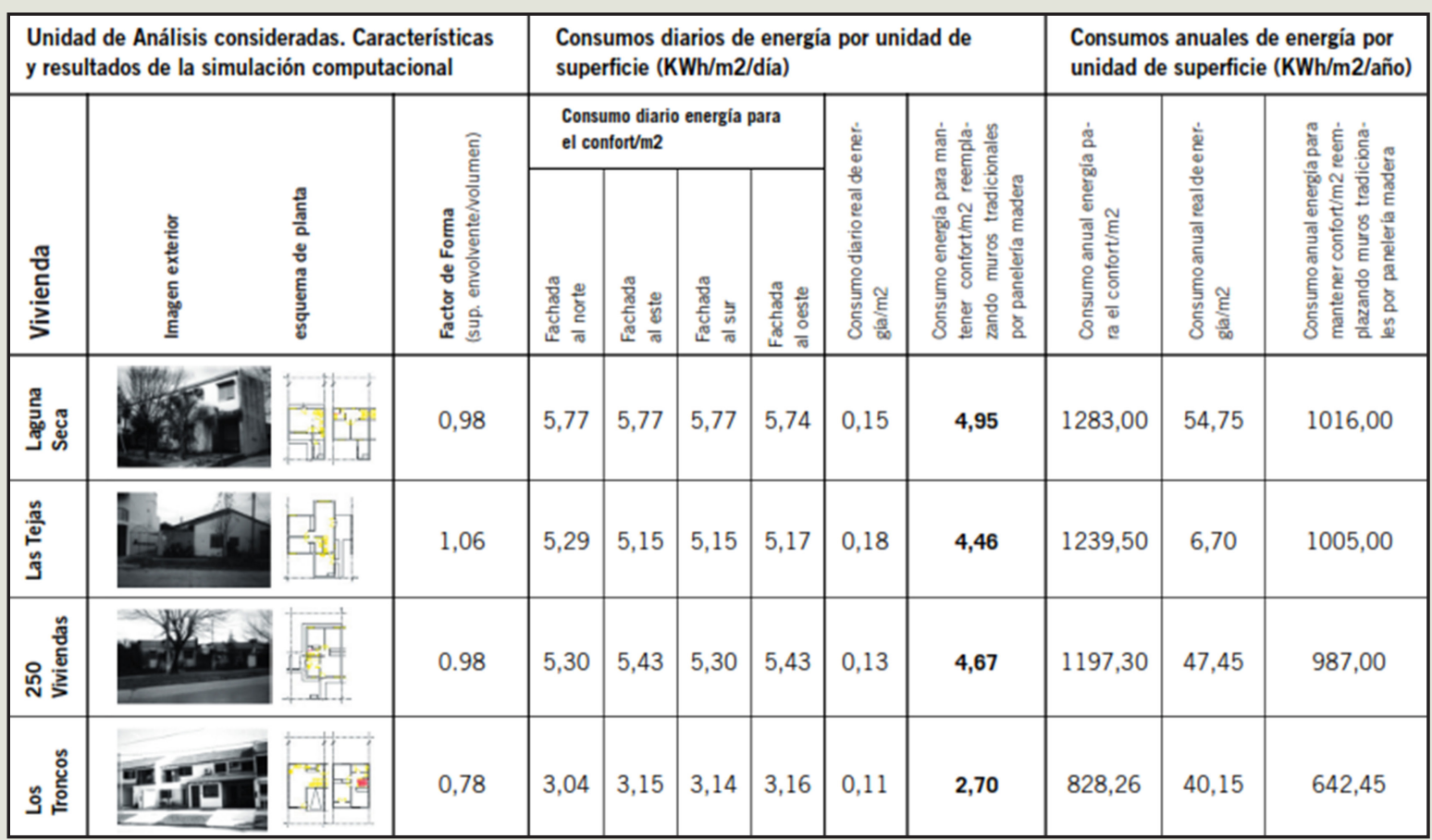

Figura 5. Planilla síntesis de los principales resultados obtenidos mediante la simulación computacional. Fuente: Alías (2003) 


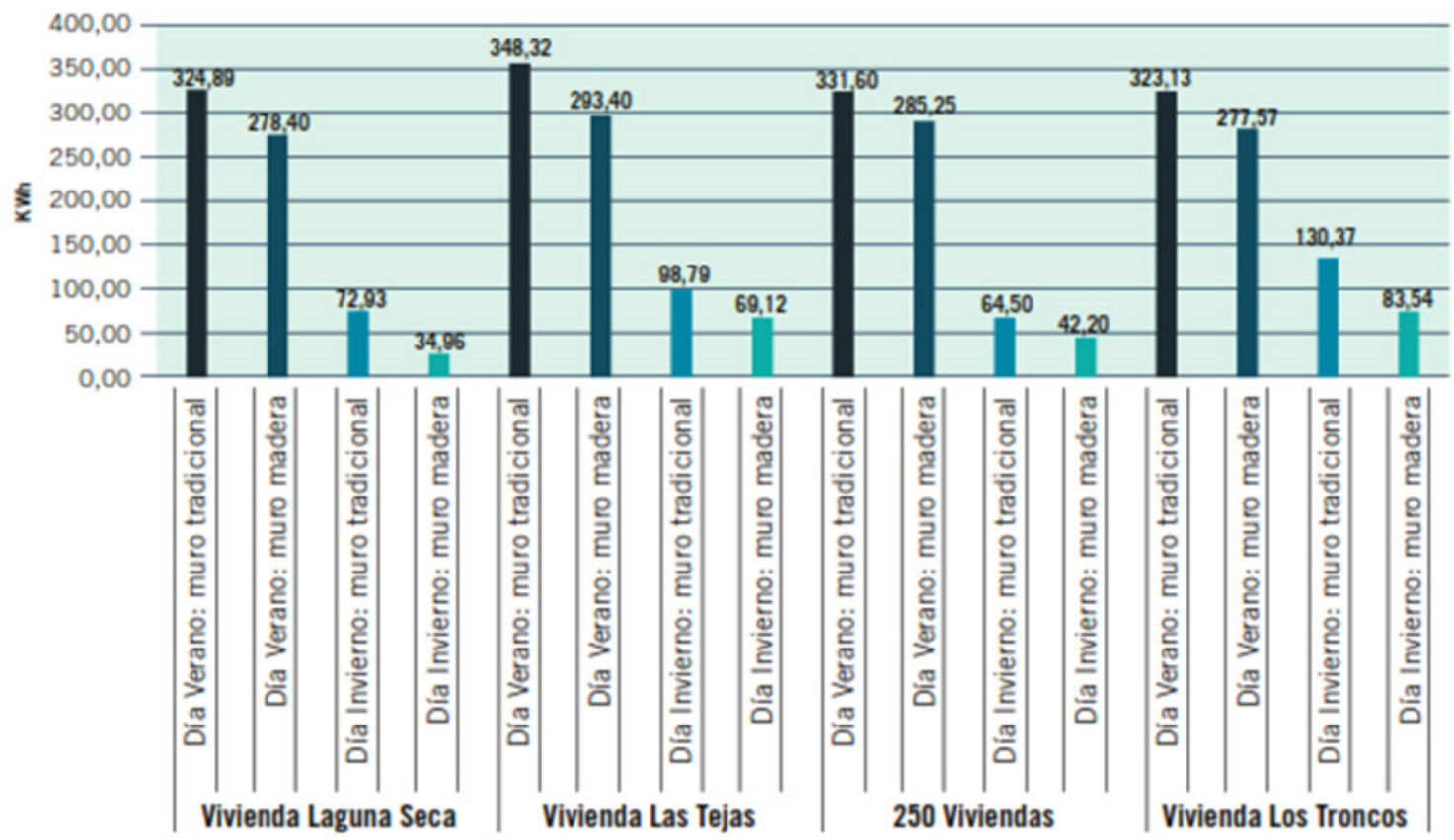

Figura 6. Comparaciones de consumos anuales por unidad de superficie: a) real; b) para mantener el confort y c) para mantener el confort con reemplazo de envolventes originales por panelería de madera. Fuente: Alías (2003)

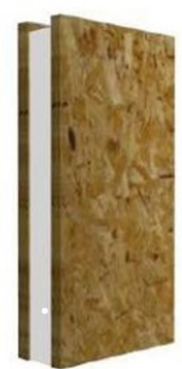

panel OSB -osb

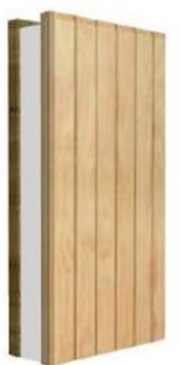

panel OSB -Terciado Ranurado

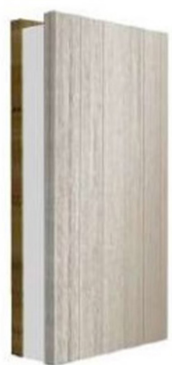

panel OSB - OSB SmartSide

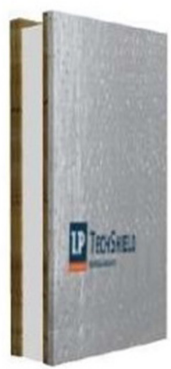

Figura 7. Pane-

les de ejemplo:

paneles tipo SIP.

Fuente: Modo Pack

Ficha de producto

y aplicación. www.

modopack.cl
Estos análisis fueron realizados comparando dos situaciones: Ia composición real de los prototipos planteados y una ficticia compuesta por un revestimiento exterior (siding de pino Elliotti impregnado de 1" y una cámara de aire débilmente ventilada dejada por las clavadoras del siding, de 1 1/2") + el panel sándwich de machimbre de pino (1/2"), poliestireno expandido (75 $\mathrm{mm}$ ) y machimbre pino (1/2") en el interior.

Figura 8. Paneles tipo SIP. Ficha técnica. Fuente: Modo Pack. Ficha de producto y aplicación. www.modopack.cl

\begin{tabular}{|c|c|c|c|}
\hline \multicolumn{4}{|c|}{ DIMENSIONES } \\
\hline $\begin{array}{c}\text { Panel SIP Estándar } \\
\text { OSB }\end{array}$ & Espesor EPS & Espesor OSB & Peso Aprox. \\
\hline $72 \mathrm{~mm}$. & $50 \mathrm{~mm} .-53 \mathrm{~mm}$ & $11.1 \mathrm{~mm} .-9.5 \mathrm{~mm}$ & $39 \mathrm{~kg}$. \\
\hline $90 \mathrm{~mm}$ & $68 \mathrm{~mm}$ & $11.1 \mathrm{~mm}$ & $40 \mathrm{~kg}$ \\
\hline $114 \mathrm{~mm}$. & $92 \mathrm{~mm}$ & $11.1 \mathrm{~mm}$ & $41 \mathrm{~kg}$. \\
\hline $120 \mathrm{~mm}$. & $98 \mathrm{~mm}$. & $11.1 \mathrm{~mm}$. & $41 \mathrm{~kg}$ \\
\hline $162 \mathrm{~mm}$. & $140 \mathrm{~mm}$. & $11.1 \mathrm{~mm}$. & $43 \mathrm{~kg}$. \\
\hline $210 \mathrm{~mm}$ & $188 \mathrm{~mm}$. & $11.1 \mathrm{~mm}$ & $45 \mathrm{~kg}$ \\
\hline Otras medidas & Consultar & Consultar & Consultar \\
\hline \multicolumn{4}{|c|}{ INFORMACIÓN TÉCNICA } \\
\hline \multicolumn{2}{|c|}{ Panel SIP Estándar OSB } & \multicolumn{2}{|c|}{ Resistencia Técnica } \\
\hline \multicolumn{2}{|c|}{$75 \mathrm{~mm}$} & \multicolumn{2}{|c|}{$1.62 \mathrm{~m}^{2}{ }^{\circ} \mathrm{c} / \mathrm{w}$} \\
\hline \multicolumn{2}{|c|}{$86 \mathrm{~mm}}$. & \multicolumn{2}{|c|}{$1.81 \mathrm{~m}^{2}{ }^{\circ} \mathrm{c} / \mathrm{w}$} \\
\hline \multicolumn{2}{|c|}{$114 \mathrm{~mm}$. } & \multicolumn{2}{|c|}{$2.40 \mathrm{~m}^{2}{ }^{\circ} \mathrm{c} / \mathrm{w}$} \\
\hline \multirow{2}{*}{\multicolumn{2}{|c|}{ Panel SIP Estándar OSB }} & \multicolumn{2}{|c|}{ RESISTENCIA } \\
\hline & & \multicolumn{2}{|c|}{ Carga admisible vertical $1298 \mathrm{Kg} / \mathrm{m}$} \\
\hline \multirow{2}{*}{\multicolumn{2}{|c|}{$86 \mathrm{~mm}}}$. & \multicolumn{2}{|c|}{ Carga admisible horizontal $382 \mathrm{Kg} / \mathrm{m}$} \\
\hline & & \multicolumn{2}{|c|}{ Carga admisible a la flexión $25 \mathrm{Kg} / \mathrm{m}$} \\
\hline
\end{tabular}




\section{CONCLUSIÓN}

Teniendo en cuenta lo estudiado en este trabajo de investigación, con la existencia de muchos antecedentes con argumentos e información fehaciente, la región del NEA sigue siendo una de las zonas que deja de lado los aspectos sustentables a la hora de hablar de la normativa de edificación. La brecha entre otras regiones y provincias, como Córdoba y Buenos Aires, es enorme en comparación con las normativas aplicadas y los sistemas de medición de sustentabilidad existentes y en funcionamiento. Además, la cuestión de la materialidad dentro de la región es un tema frágil, ya que ninguna normativa rige o se articula con la construcción en seco. Este sistema, relativamente nuevo dentro del país, es altamente eficiente, rápido, económico y limpio. Debemos plantearnos esta forma de construir como una alternativa a lo ya existente.

Debemos abordar estos temas con carácter urgente, no solo por la característica climática de la región, sino porque el NEA está en pleno crecimiento y desarrollo y, a su vez, es una de las regiones más vulnerables del país. Estamos a tiempo de realizar cambios contundentes. Sin duda alguna, tenemos que tomar riendas sobre la cuestión para dar un giro drástico a los aspectos más críticos de este sistema: la habitabilidad y la demanda energética.

\section{REFERENCIAS BIBLIOGRÉFICAS}

Alías, H. (2003). Estudio de la eficiencia ambiental del uso de madera en la construcción de viviendas en el NEA en base al análisis energético y de Ciclo de Vida. (Tesis de maestría). Maestría en Gestión Ambiental de la Facultad de Arquitectura y Urbanismo de la Universidad Nacional del Nordeste. Resistencia, Chaco, Argentina

Anexo - Ley N. ${ }^{\circ} 6100$ (2018). Código de edificación de la Ciudad de Buenos Aires. Buenos Aires, Argentina. Blasco Lucas, I. (2008). Aportes de la arquitectura sustentable en e sector residencial sobre el balance energético ambiental argentino. Avances en Energías Renovables y Medio Ambiente. Vol. 12. Argentina. Pp. 07.17-07.24.

De Schiller, S.; Gomes Da Silva, V.; Goijberg, N. \& Treviño, C. (2003) Edificación sustentable: consideraciones para la calificación del hábitat construido en el contexto regional latinoamericano. Avances en Energías Renovables y Medio Ambiente 7, pp. 13-05.18.

INDEC (2007). Estadísticas Demográficas: Censos y Proyecciones. http://www.indec.mecon.gov.ar/

Instituto de la Vivienda de la Provincia de Buenos Aires. Acondicionamiento higrotérmico de edificios. Manual de aplicación Ley 13059. Buenos Aires, Argentina.

Instituto de Sustentabilidad - Co- legio de Arquitectos de la Provincia de Córdoba. Etiquetación de sustentabilidad edilicia. Protocolo eSe. Marco de referencia.

Jacobo, G. \& Alías, H. (2007). Construcción sostenible. Materiales de construcción energética y ambientalmente eficientes en el nordeste de Argentina. Resistencia. Chaco, Argentina.

Normas IRAM 11601, 11603, 11604, 11605, 11625, 11630, 11659, 11900. Ordenanza N. ${ }^{\circ} 8757$ - Concejo Municipal de Rosario: incorporación al Reglamento de Edificación de la Ciudad, sección: Aspectos Higrotérmicos y Eficiencia Energética de las construcciones. Rosario. Santa Fe, Argentina

Romano Pamies, C.; Alías, H. \& Jacobo, G. (2018). Lineamientos e indicadores para la valoración de la sustentabilidad edilicia, de factible incorporación a las normativas de edificación vigentes en Resistencia y Corrientes. Resistencia. Chaco, Argentina.

Revista Vivienda (2014,04 de febrero). Ladrillo vs. Construcción en seco: derribando el mito. Revista vivienda. http://www.revistavivienda.com.ar/ actualidad/gacetillas/ladrillos-vsconstruccion-en-seco-derribandoel-mito

Suárez, Edgardo F. (2017). Etiquetado en Viviendas - Iniciativas en provincia de Córdoba. [Ponencia]

Jornada Nacional de Etiquetado en Eficiencia Energética de Viviendas. https://scripts.minem.gob.ar/octopus $/$ archivos . php?file $=7572$ 